\title{
低渗煤层 $\mathrm{CO}_{2}$ 预裂增透高效瓦斯抽采原理及应用
}

汪虎

陕西涁长胡家河矿业有限公司

DOI:10.32629/gmsm.v2i4.247

[摘 要] 要想有效提高低渗透煤层抽采效率、缩短抽采时间, 就需要使用 $\mathrm{CO} 2$ 高能气体预裂增透技术来对低渗透煤层进行改 造, 以此来保证煤层的透气性,进一步提升瓦斯的抽采量与抽采效率。基于此, 本文首先将分析高能气体欲裂增透瓦斯抽采技术 的原理,并在此基础上阐述它的具体应用以及效果。

[关键词] 低渗透煤层; $\mathrm{CO}_{2}$ 预裂; 卸压增透; 瓦斯抽采

\section{1 高能气体预裂增透瓦斯抽采技术原理}

1. 1 高能气体预裂原理

一般来说, 在密闭空间液气两相转变过程中, 高能气体 预破碎和防渗透技术需要通过释放大量高能气体来预裂煤 体。当温度低于 31 摄氏度, 压力是 7.2 兆帕的时候, $\mathrm{CO}_{2}$ 以液 态的形式存在, 但是, 在温度大于 31 摄氏度的时候, 不管压 力的数值是多少, 液态的 $\mathrm{CO}_{2}$ 都会变为气态。按照这个原理, 在压裂管中采用一种特殊的高压泵来填充 $\mathrm{CO}_{2}$ 液体。在预裂 的过程中, 需要接通加热器的电流, 并通过内部化学物质的 快速放热反应, 使得 $\mathrm{CO}_{2}$ 液体可以在 20 到 40 毫秒以内快速 地变成气态形式, 在这个过程中, 它的体积会膨胀到原来的 六百倍左右, 压力也会提高到设定的压力, 剪切片破裂, 这时 高能气体就会立刻由压裂管喷气阀喷射而出, 因为喷射而产 生的流动射流与后期气体膨胀形成的静态压力会对媒体产 生共同的作用力, 然后就会导致媒体裂隙的产生。具体如下 图 1 所示:

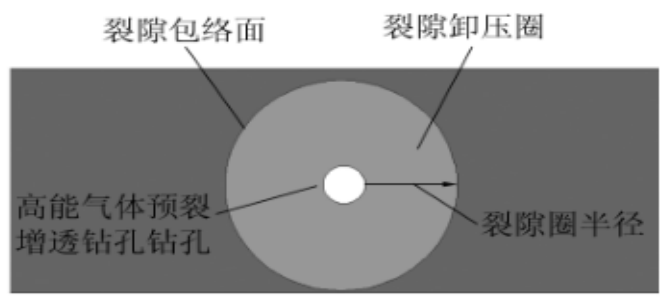

(a) 裂隙卸压圈示意

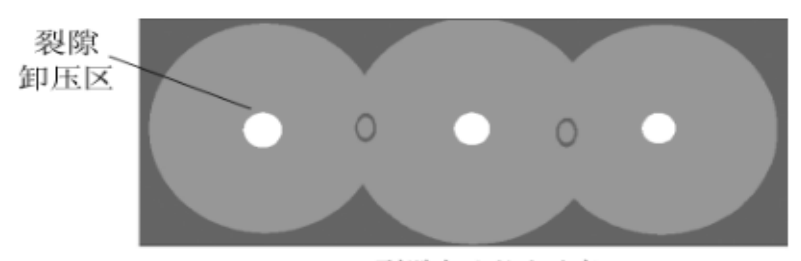

(b) 裂隙卸压区示意

图 1 高能气体预裂增透技术机理示意

1.2 卸压增透高效抽采技术原理

高能气体预裂增透瓦斯抽采技术主要通过高能气体预 裂增加煤层透气性, 并加快煤体内吸附态瓦斯的快速解吸, 从而可以提高瓦斯的抽采量与抽采效率, 缩短抽采的时间, 具体的作用原理有以下几点: 第一, 高能气体预裂在增透过
程中, 由高压气体所造成的射流与静压力会对煤体产生一个 作用力, 从而导致煤体裂隙, 也会导致煤体内部那些已经被 填充和压实的裂缝再次打开并不断扩大。在使用单孔和多孔 高能气体预裂增透的过程中, 会在煤层内部形成单个裂隙卸 压区, 在这个区域内部煤体裂隙数量与规模的不断扩大, 会 造成瓦斯运移通道的提高, 有效改善应力集中的问题, 然后 就会提高区域内煤层的透气性。第二, 在高能气体预裂增透 的时候, 干燥的高压气体会对煤体产生作用, 所以煤层内部 并不会有额外的积水出现, 从而也就可以防止因大量水的侵 入导致煤层的含水饱和度提高, 而且也能够防止因水所反应 的产生影响瓦斯的抽采速度与效率。

\section{2 现场应用及分析}

2.1 试验区域概况

E1206工作面与E2202工作面位于某个试验区域, 目前 需要开采 2 号煤层, 煤层的平均厚度介于 6 米到 7 米之间, 倾 角介于 3 度到 15 度之间。在该试验区域中, 2 号煤层的瓦斯含 量是相对比较高的, 在实验室对其进行测量之后发现, 瓦斯 的含量是 5.56 到 $15.01 \mathrm{~m}^{3} / \mathrm{t}$, 平均的含量大概是 $12.6 \mathrm{~m}^{3} / \mathrm{t}$ 。

2.2 掘进工作面预裂增透瓦斯抽采

2.2.1 设计施工情况

按照工作面的布置情况, 将 E2202 进风巷作为实验区。 按照掘进面的布置方案, 在掘进工作面开一个预裂孔, 并在 掘进工作面的左右两面钻场内分别开五个辅助的瓦斯抽采 钻孔, 具体的布置方式与参数如下图 2 与下表 1:

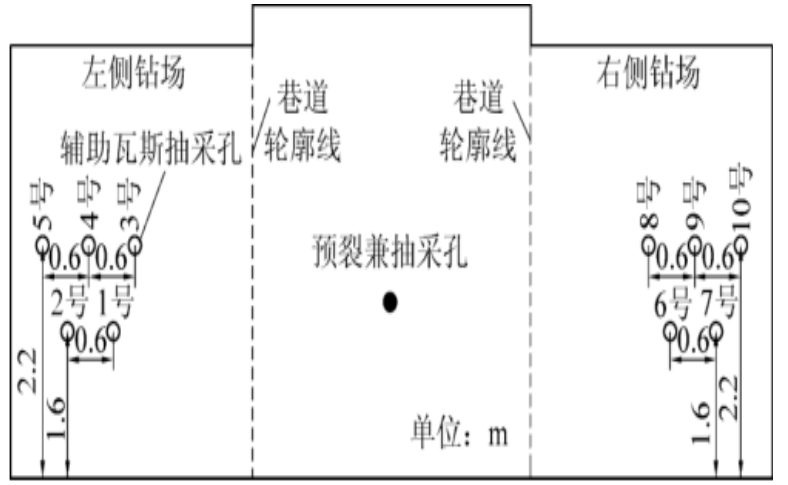

图 2 掘进工作面高能气体预裂增透钻孔布置 


\begin{tabular}{|c|c|c|c|}
\hline \multicolumn{2}{|c|}{ 工作南智 } & 掘进工作面 & 回来工作西 \\
\hline \multirow{4}{*}{ 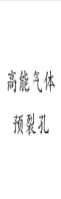 } & 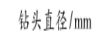 & 94 & 94 \\
\hline & 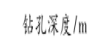 & 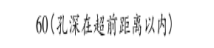 & 》1/3工作西究度 \\
\hline & 批 & 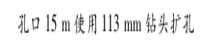 & 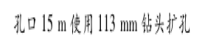 \\
\hline & 战礼解 & 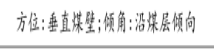 & 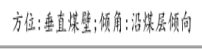 \\
\hline \multirow{4}{*}{ 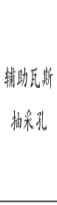 } & 击头直隻/mm & 94 & 94 \\
\hline & 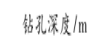 & 80 & $\geqslant 1 / 3$ 工作西究度 \\
\hline & 䖪行解 & 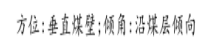 & 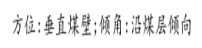 \\
\hline & 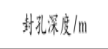 & $10-15$ & 10015 \\
\hline \multicolumn{2}{|c|}{ 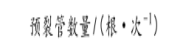 } & $15-20$ & $15 \% 20$ \\
\hline \multicolumn{2}{|c|}{ 赫废力 } & $5 \times 7$ & $5 \mu 7$ \\
\hline
\end{tabular}

\subsection{2 应用效果分析}

首先需要在E2202进风巷的左右钻场分别开五个起辅 助作用的瓦斯抽采钻孔, 并网进行抽采, 并在掘进工作面开 一个预裂孔用于高能气体预裂增透。因为会对瓦斯抽采效 果产生影响的因素有很多个, 单独的某个钻孔可能会因为 一些特殊因素的影响而难以体现出真正的预裂增透效果, 所以这次实验并不是单纯地针对某个抽采钻孔的效果来展 开观察, 只是对掘进工作面预裂区域里钻孔的整体抽采情 况进行观察与分析。所以, 把所有的钻孔连接在同一个汇流 管上并入抽采支管, 然后在汇流管上安装孔板流量计, 以此 来实现对掘进工作面钻孔整体抽采情况的监控与分析。在 预裂结束抽采 20 天以后, 平均的抽采纯流量是 $0.75 \mathrm{~m}^{3} / \mathrm{min}$, 平均百米钻孔抽采纯瓦斯流量为 $0.1317 \mathrm{~m}^{3} /(\mathrm{min} \cdot \mathrm{hm})$ 。对 比E2202进风巷的抽采数据和预裂之前的数据, 结果如下表 2所示:
表 2 掘进工作面预裂前后抽采数据对比

\begin{tabular}{|c|c|c|c|}
\hline 抽采参数 & 预裂前 & 预裂后 & 增幅 $/ \%$ \\
\hline 平均瓦斯体积分数 $/ \%$ & 21.2 & 52 & 145 \\
\hline $\begin{array}{c}\text { 平均抽采混合流量/ } \\
\left(\mathrm{m}^{3} \cdot \mathrm{min}^{-1}\right)\end{array}$ & 0.62 & 1. 43 & 131 \\
\hline $\begin{array}{c}\text { 平均抽采纯流量/ } \\
\left(\mathrm{m}^{3} \cdot \mathrm{min}^{-1}\right)\end{array}$ & 0. 13 & 0.74 & 469 \\
\hline $\begin{array}{c}\text { 平均百米钻孔抽采纯流量/ } \\
\left(\mathrm{m}^{3} \cdot \mathrm{min}^{-1} \cdot \mathrm{hm}^{-1}\right)\end{array}$ & 0.0070 & 0.1217 & 1781 \\
\hline
\end{tabular}

由表 2 中的数据能够得出, 在掘进工作面开展高能气体 预裂增透以后, 可以有效提高抽采钻孔的抽采浓度、混合流 量以及纯瓦斯流量。

\section{3 结束语}

在密闭空间液气两相转化过程中产生的高压气体, C02预 裂产生的高压气体会对煤体造成破坏, 从而就会导致很多新 裂隙的产生, 而且也会导致之前的裂缝不断扩大, 最终就会形 成裂隙卸压区。在这个区域内, 煤层的透气性不断提高, 瓦斯 的运移阻力减少, 区域内的煤层瓦斯解吸率提高, 许多吸附态 瓦斯会演变成游离的状态, 瓦斯运移的驱动力进一步提高, 从 而也就可以提高煤层的透气性以及瓦斯的抽采效率。

\section{[参考文献]}

[1]郭臣业,沈大富,张翠兰.煤矿井下控制水力压裂煤层增透 关键技术及应用[J].煤炭科学技术,2015,43(2):114-118+122.

[2]申宝宏,刘见中, 雷毅.我国煤矿区煤层气开发利用技 术现状及展望[J].煤炭科学技术,2015,43(2):1-4.

[3]付江伟.井下水力压裂煤层应力场与瓦斯流场模拟研 究[D].中国矿业大学,2013,(05):179. 